International Journal of Engineering \& Technology, $7(3.31)(2018) 115-120$
International Journal of Engineering \& Technology
SPC
Website: www.sciencepubco.com/index.php/IJET
Research paper

\title{
Controllability, Observability and Stability of Volterra Type Non-Linear Matrix Integro-Dynamic System on Time Scales
}

\author{
G.V. Ramana ${ }^{1}$, G.V. S. R. Deekshitulu ${ }^{2 *}$ \\ ${ }^{1}$ Department of Mathematics, \\ Aditya Engineering College, Surampalem, A.P., India. \\ ${ }^{2}$ Department of Mathematics, \\ UCEK, JNTUK, Kakinada, A.P., India. \\ *Corresponding author E-mail: dixitgvsr@hotmail.com
}

\begin{abstract}
This paper investigates the controllability, observability and stability of the solution of Volterra type non linear matrix integro dynamic system on time scales.
\end{abstract}

Keywords: Controllability; non-linear Volterra type matrix integro-dynamic system; observability; stability; time scales.

\section{Introduction}

In many engineering problems, one may desire to have a system that follows a preassigned behaviour. In other words, necessary steps have to be taken to avoid unwanted behaviour in a system and to compel the system to follow a desired behaviour. The origin of control theory stems from determining these steps called controls. After R.E. Kalman introduced general control theory in 1960 , many engineers and mathematicians got attracted by this theory [7, $9,10,11,12,13,16]$. The importance of control theory in mathematics and its applications in diverse areas such as adoptive controls [9], communication networks [10], switching systems [14], dynamic programs [15], are well established.

The theory of time scales, introduced by Hilger $[3,8]$ at the end of the twentieth century as a means to unify the difference and the differential calculus, is now a well-established subject.

In [7], J.M. Davis, Ian A. Gravagne, Billy J. Jackson, R.J. Marks discussed the controllability, observability realizability and stability of linear dynamic system on time scales.

On the other hand, the theory of Volterra integro-dynamic equation has drawn the attention of many mathematicians in the last decade [6]. In [1], Adivar derived principal matrix solution using variation of parameters formula for integro-dynamic equations on time scales. In [2], Becker investigated the solution using variation of parameters formula for a integro-dynamic equations and its adjoint. Burton and Mahfoud discussed the various stability properties of integro-dynamic equations in $[4,5,6]$.

Controllability, observability and stability of Volterra integro dynamic system on time scales were studied Awais Yonus and Ghaus ur Rahman [16] . They considered linear integro dynamic system of the form
$x^{\Delta}(t)=A(t) x(t)+\int_{t_{0}}^{t} K(t, s) x(s) \Delta s+B(t) u(t)$.

Anyhow, much of contribution on controllability, observability and stability of non-linear integro dynamic systems on time scales is not available in literature. With this motivation, in this paper, we establish some new results on controllability, observability and stability of non linear matrix integro dynamic system on time scales.

\section{Preliminary Results}

Throught this paper $\mathbb{T}$ denotes time scale(an arbitrary nonempty closed subset of the real numbers).

Definition 2.1: ([3]) The mappings $\sigma$ and $\rho: \mathbb{T} \rightarrow \mathbb{R}$ where $\mathbb{T}$ is any closed subset of reals, are defined as $\sigma(t)=\inf \{s \in \mathbb{T}: s>t\}$ and $\rho(t)=\sup \{s \in \mathbb{T}: s<t\}$.

Definition 2.2:([3]) A non-maximal element $\mathrm{t}$ in $\mathbb{T}$ is called right dense if $\sigma(t)=t$; right scattered if $\sigma(t)>t$; left dense if $\rho(t)=t$ and left scattered if $\rho(t)<t$.

Definition 2.3:([3]) If $\mathbb{T}$ has a left scattered maximum $M$, then $\mathbb{T}^{k}=\mathbb{T}-\{M\}$, otherwise, $\mathbb{T}^{k}=\mathbb{T}$. If $\mathbb{T}$ has a right scattered minimum $m$, then $\mathbb{T}^{k}=\mathbb{T}-\{m\}$, otherwise, $\mathbb{T}^{k}=\mathbb{T}$.

Definition 2.4:([3]) The function $\mu^{*}: \mathbb{T}^{k} \rightarrow \mathbb{R}^{+}$defined by $\mu^{*}(t)=\mu(\sigma(t), t)$ for $t \in \mathbb{T}$ is called graininess. If $t$ is right dense, then $\mu^{*}=0$ and if $t$ is right scattered, then $\mu^{*}=\sigma(t)-t$.

Definition 2.5:([3]) A mapping $f: \mathbb{T} \rightarrow \mathbb{R}$ is said to be differentiable at $t \in \mathbb{T}^{k}$, if there exists an $\alpha \in \mathbb{R}$ such that for any $\varepsilon>0$ there exists a neighbourhood $Q$ of $t$ satisfying $\mid f(\sigma(t))-$ 
$f(s)-(\sigma(t)-s) \alpha|\leq \varepsilon| \sigma(t)-s \mid$ for all $s \in Q$.

Definition 2.6:([3]) An $(n \times n)$ matrix valued function $A$ on a time scale $\mathbb{T}$ is called regressive provided

is invertible for all $t \in \mathbb{T}^{k}$.

$$
I+\mu(t) A(t)
$$

Theorem 2.7:([3]) Assume $f: \mathbb{T} \rightarrow \mathbb{R}$ is a function and let $t \in \mathbb{T}^{k}$. Then we have the following

(a) If $f$ is differentiable at $t$, then $f$ is continuous at $t$.

(b) If $f$ is continuous at $t$ and $t$ is right-scattered, then $f$ is differentiable at $t$ with

$$
f^{\Delta}(t)=\frac{f(\sigma(t))-f(t)}{\mu(t)} .
$$

(c) If $t$ is right-dense, then $f$ is differentiable at $t$ iff the limit

$$
\lim _{s \rightarrow t} \frac{f(t)-f(s)}{t-s} .
$$

exists as a finite number. In this case

(d) If $f$ is differentiable at $t$, then

$$
f^{\Delta}(t)=\lim _{s \rightarrow t} \frac{f(t)-f(s)}{t-s} .
$$

$$
f(\sigma(t))=f(t)+\mu(t) f^{\Delta}(t) .
$$

Theorem 2.8:([3]) Suppose $A, B$ and $C$ are differentiable $(n \times n)$ matrix-valued functions. Then
(a) $(A+B)^{\Delta}=A^{\Delta}+B^{\Delta}$
(b) $(\alpha A)^{\Delta}=\alpha A^{\Delta}$ if $\alpha$ is constant;
(c) $(A B)^{\Delta}=A^{\Delta} B^{\sigma}+A B^{\Delta}=A^{\sigma} B^{\Delta}+A^{\Delta} B$;
(d) $(A B C)^{\Delta}=A^{\Delta} B C+A^{\sigma} B^{\Delta} C^{\sigma}+A^{\sigma} B C^{\Delta}=A^{\Delta} B C+A^{\sigma} B^{\Delta} C+$ $A^{\sigma} B^{\sigma} C^{\Delta}$

Lemma 2.9: ([3]) Let $y \in C_{r d}(\mathbb{T}, \mathbb{R}), p \in \mathbb{R}^{+}, p \geq 0$ and $\alpha \in \mathbb{R}$. Then

for all $t \in \mathbb{T}$, implies

$$
y(t) \leq \alpha+\int_{t_{0}}^{t} y(s) p(s) \Delta s
$$

for all $t \in \mathbb{T}$.

$$
y(t) \leq \alpha e_{p}\left(t, t_{0}\right)
$$

Lemma 2.10: ([3]) Let $\alpha \in \mathbb{R}$ with $\alpha \in \mathbb{R}^{+}$. Then $e_{\alpha}(t, s) \geq 1+\alpha(t-s)$ for all $t \geq s$

\section{Main Results}

In this paper, a non linear integro dynamic system on time scale $\mathbb{T}=[0, \infty)$ of the form

$$
\left.\begin{array}{ll}
X^{\Delta}(t) & =A(t) X(t)+\left(\int_{t_{o}}^{t} K(t, s, X(s)) \Delta s+B(t)\right) U(t), \\
Z(t) & =C(t) X(t), \\
X\left(t_{0}\right) & =X_{0} .
\end{array}\right\}
$$

is considered. Here $t_{0} \in \mathbb{T}^{k}, X \in \mathbb{R}^{n \times n}, U \in \mathbb{R}^{n \times n}$. The matrix functions $A(t), K(t, s, X(s)), B(t)$ and $C(t)$ are all $(n \times n)$ continuous and regressive with respect to their arguments on $\mathbb{T}$.

Theorem 3.1: Let $A(t), \mathrm{B}(\mathrm{t})$ and $\mathrm{K}(\mathrm{t}, \mathrm{s}, \mathrm{X}(\mathrm{s}))$ be continuous and regressive $(n \times n)$ matrices. Then the solution of (1) is given by

$$
\begin{aligned}
X(t)= & \Psi\left(t, t_{0}\right) X_{0} \\
+ & \Psi\left(t, t_{0}\right) \int_{t_{0}}^{t} \Psi\left(t_{0}, \sigma(\tau)\right)\left(\int_{t_{0}}^{\tau} K(\tau, s, X(s)) \Delta s+B(\tau)\right) \\
& \times U(\tau) \Delta \tau
\end{aligned}
$$

where $\Psi\left(t, t_{0}\right)$ is state transition matrix and is given by

$\Psi\left(t, t_{0}\right)=\Phi(t) \Phi^{-1}\left(t_{0}\right)$ and $\Phi(t)$ is a fundamental matrix of
$X^{\Delta}(t)=A(t) X(t)$

Proof:

Let $\Phi(t)$ be the fundamental matrix of

$X^{\Delta}(t)=A(t) X(t)$.

Then any solution of (3.3) is of the form

$$
X(t)=\Phi(t) C \text {. }
$$

where $C$ is a constant matrix. Let the particular solution $\bar{X}(t)$ of (1) be of the form

Then we have

$$
\bar{X}(t)=\Phi(t) R(t)
$$

$\Phi^{\Delta}(t) R(t)+\Phi(\sigma(t)) R^{\Delta}(t)=A(t) \Phi(t) R(t)$

$$
\begin{gathered}
+\left(\int_{t_{0}}^{t} K(t, s, X(s)) \Delta s+B(t)\right) U(t) \\
R^{\Delta}(t)=\Phi^{-1}(\sigma(t))\left(\int_{t_{0}}^{t} K(t, s, X(s)) \Delta s+B(t)\right) U(t) .
\end{gathered}
$$

Integration on both sides between the limits $t_{0}$ to $t$, we have

$$
R(t)=\int_{t_{0}}^{t} \Phi^{-1}(\sigma(\tau))\left(\int_{t_{0}}^{\tau} K(\tau, s, X(s)) \Delta s+B(\tau)\right) U(\tau) \Delta \tau .
$$

and the general solution is given by

$$
\begin{aligned}
X(t)= & \Phi(t) C \\
& +\Phi(t) \int_{t_{0}}^{t} \Phi^{-1}(\sigma(\tau))\left(\int_{t_{0}}^{\tau} K(\tau, s, X(s)) \Delta s+B(\tau)\right) U(\tau) \Delta \tau .
\end{aligned}
$$

Clearly, $C=\Phi^{-1}\left(t_{0}\right) X_{0}$

Hence the general solution of (1) is (2)

Definition 3.1:([7,16]) The regressive non-linear system (1) is said to be completely controllable on $\left[t_{0}, t_{f}\right]$ if for any initial time $t_{0}$, initial state $X\left(t_{0}\right)=X_{0}$ and given final state $X_{f}$, there exists a finite time $t_{f}>t_{0}$ and a rd-continuous control $U(t)$ for $t_{0} \leq t \leq$ $t_{f}$ such that $X\left(t_{f}\right)=X_{f}$.

Theorem 3.2: The system (1) is completely controllable if and only if the $(n \times n)$ symmetric controllability Gramian matrix given by $G_{c}\left(t_{0}, t_{f}\right)=\int_{t_{0}}^{t_{f}} \Psi\left(t_{0}, \sigma(\tau)\right) H(\tau, X(\tau)) H^{T}(\tau, X(\tau)) \Psi^{T}\left(t_{0}, \sigma(\tau)\right) \Delta \tau$ is invertible, where

$$
H(\tau, X(\tau))=\int_{t_{0}}^{\tau} K(\tau, s, X(s)) \Delta s+B(\tau) .
$$

Proof:

Suppose that Gramian matrix $G_{c}\left(t_{0}, t_{f}\right)$ is invertible. Then for given $X_{0}$ and $X_{f}$, choose continuous matrix function $U(t)$ for $t \in\left[t_{0}, t_{f}\right)$ as

$U(t)=-H^{T}(t, X(t)) \Psi^{T}\left(t_{0}, \sigma(t)\right) G_{c}^{-1}\left(t_{0}, t_{f}\right)\left[X_{0}-\Psi\left(t_{0}, t_{f}\right) X_{f}\right]$ and extend $U(t)$ continuously for all other values of $t$. The corresponding solution of the system (1) at $t=t_{f}$ can be written as 


$$
\begin{aligned}
& X\left(t_{f}\right)=\Psi\left(t_{f}, t_{0}\right) X_{0} \\
& +\Psi\left(t_{f}, t_{0}\right) \int_{t_{0}}^{t_{f}} \Psi\left(t_{0}, \sigma(\tau)\right)\left(\int_{t_{0}}^{\tau} K(\tau, s, X(s)) \Delta s+B(\tau)\right) \\
& \times U(\tau) \Delta \tau \\
& =\Psi\left(t_{f}, t_{0}\right) X_{0}+\Psi\left(t_{f}, t_{0}\right) \int_{t_{0}}^{t_{f}} \Psi\left(t_{0}, \sigma(\tau)\right) H(\tau, X(\tau)) \\
& \left\{-H^{T}(\tau, X(\tau)) \Psi^{T}\left(t_{0}, \sigma(\tau)\right) G_{c}^{-1}\left(t_{0}, t_{f}\right)\right. \\
& \left.\times\left[X_{0}-\Psi\left(t_{0}, t_{f}\right) X_{f}\right]\right\} \Delta \tau \\
& =\Psi\left(t_{f}, t_{0}\right) X_{0}-\Psi\left(t_{f}, t_{0}\right) G_{c}\left(t_{0}, t_{f}\right) G_{c}^{-1}\left(t_{0}, t_{f}\right) \\
& \times\left[X_{0}-\Psi\left(t_{0}, t_{f}\right) X_{f}\right] \\
& =\Psi\left(t_{f}, t_{0}\right) X_{0}-\Psi\left(t_{f}, t_{0}\right)\left[X_{0}-\Psi\left(t_{0}, t_{f}\right) X_{f}\right] \\
& =\Psi\left(t_{f}, t_{0}\right) X_{0}-\Psi\left(t_{f}, t_{0}\right) X_{0}+X_{f} \\
& =X_{f} \text {. }
\end{aligned}
$$

Conversely, suppose that the system be controllable. If $G_{c}\left(t_{0}, t_{f}\right)$ is not invertiable then there exists a constant vector $Y \neq 0$ such that

$$
\begin{aligned}
0 & =Y^{T} G_{c}\left(t_{0}, t_{f}\right) Y \\
& =\int_{t_{0}}^{t_{f}} Y^{T} \Psi\left(t_{0}, \sigma(\tau)\right) H(\tau, X(\tau)) H^{T}(\tau, X(\tau)) \Psi^{T}\left(t_{0}, \sigma(\tau)\right) Y \Delta \tau \\
& =\int_{t_{0}}^{t_{f}}\left\|Y^{T} \Psi\left(t_{0}, \sigma(\tau)\right) H(\tau, X(\tau))\right\|^{2} \Delta \tau
\end{aligned}
$$

and hence $Y^{T} \Psi\left(t_{0}, \sigma(\tau)\right) H(\tau, X(\tau))=0$, for all $\tau \in\left[t_{0}, t_{f}\right]$. Since the system is controllable on $\left[t_{0}, t_{f}\right]$ and choose $X\left(t_{0}\right)=Y+\Psi\left(t_{0}, t_{f}\right) X_{f}$, then there exists a $U(\tau)$ such that

$$
\begin{aligned}
X_{f}= & \Psi\left(t_{f}, t_{0}\right) X_{0} \\
+ & \Psi\left(t_{f}, t_{0}\right) \int_{t_{0}}^{t_{f}} \Psi\left(t_{0}, \sigma(\tau)\right)\left(\int_{t_{0}}^{\tau} K(\tau, s, X(s)) \Delta s+B(\tau)\right) \\
& \times U(\tau) \Delta \tau .
\end{aligned}
$$

Or

$$
\begin{aligned}
\Psi\left(t_{0}, t_{f}\right) X_{f} & =X_{0}+\int_{t_{0}}^{t_{f}} \Psi\left(t_{0}, \sigma(\tau)\right) H(\tau, X(\tau)) U(\tau) \Delta \tau \\
X_{0}-Y & =X_{0}+\int_{t_{0}}^{t_{f}} \Psi\left(t_{0}, \sigma(\tau)\right) H(\tau, X(\tau)) U(\tau) \Delta \tau \\
Y^{T} Y & =-\int_{t_{0}}^{t_{f}} Y^{T} \Psi\left(t_{0}, \sigma(\tau)\right) H(\tau, X(\tau)) U(\tau) \Delta \tau \\
Y^{T} Y & =0 \\
\Rightarrow Y & =0 .
\end{aligned}
$$

Which is contradiction to $Y \neq 0$, and hence $G_{c}\left(t_{0}, t_{f}\right)$ is invertible.

Definition 3.2: ([7, 16]) The regressive non-linear system (1) is said to be completely observable on $\left[t_{0}, t_{f}\right]$ if any initial time $t_{0}$ and initial state $X\left(t_{0}\right)=X_{0}$, there exists a finite time $t_{f}>t_{0}$ such that the knowledge of the control $U(t)$ and output $Z(t)$ for $t_{0} \leq t \leq t_{f}$ is sufficient to determine $X_{0}$ uniquely.

Theorem 3.3: The system (1) is completely observable if and only if the $(n \times n)$ symmetric observability Gramian matrix given by

$$
G_{o}\left(t_{0}, t_{f}\right)=\int_{t_{0}}^{t_{f}} \Psi^{T}\left(t, t_{0}\right) C^{T}(t) C(t) \Psi\left(t, t_{0}\right) \Delta t
$$

is invertible

Proof:

Suppose that observability Gramian matrix $G_{o}\left(t_{0}, t_{f}\right)$ is invertible. Without loss of generality, assume that the control $U(t)=0$ for $t \in\left[t_{0}, t_{f}\right]$. Then the solution of (1) is

and out put

$$
X(t)=\Psi\left(t, t_{0}\right) X_{0}
$$

$$
Z(t)=C(t) \Psi\left(t, t_{0}\right) X_{0} .
$$

Multiplying the above equation with $\Psi^{T}\left(t, t_{0}\right) C^{T}(t)$ and integration on both sides between the limits $t_{0}$ to $t_{f}$, we have

$$
\begin{gathered}
\int_{t_{0}}^{t_{f}} \Psi^{T}\left(t, t_{0}\right) C^{T}(t) C(t) \Psi\left(t, t_{0}\right) X_{0} \Delta t=\int_{t_{0}}^{t_{f}} \Psi^{T}\left(t, t_{0}\right) C^{T}(t) Z(t) \Delta t \\
G_{o}\left(t_{0}, t_{f}\right) X_{0}=\int_{t_{0}}^{t_{f}} \Psi^{T}\left(t, t_{0}\right) C^{T}(t) Z(t) \Delta t
\end{gathered}
$$

or

$$
X_{0}=G_{o}^{-1}\left(t_{0}, t_{f}\right) \int_{t_{0}}^{t_{f}} \Psi^{T}\left(t, t_{0}\right) C^{T}(t) Z(t) \Delta t .
$$

It follows that $X_{0}$ is uniquely determined with the knowledge of $Z(t)$ when $U(t)=0$ for $t \in\left[t_{0}, t_{f}\right]$. Therefore the system (1) is observable.

Conversely, suppose that $G_{o}\left(t_{0}, t_{f}\right)$ is not invertible and the system (1) is observable. This implies that there exists a constant vector $Y \neq 0$ such that

$$
\begin{aligned}
0 & =Y^{T} G_{o}\left(t_{0}, t_{f}\right) Y \\
& =\int_{t_{0}}^{t_{f}} Y^{T} \Psi^{T}\left(t, t_{0}\right) C^{T}(t) C(t) \Psi\left(t_{0}, t\right) Y \Delta t \\
& =\int_{t_{0}}^{t_{f}}\left[C(t) \Psi\left(t, t_{0}\right) Y\right]^{T}\left[C(t) \Psi\left(t, t_{0}\right) Y\right] \Delta t \\
& =\int_{t_{0}}^{t_{f}}\left\|C(t) \Psi\left(t, t_{0}\right) Y\right\|^{2} \Delta t
\end{aligned}
$$

and hence $C(t) \Psi\left(t, t_{0}\right) Y=0, t \in\left[t_{0}, t_{f}\right]$. If $X_{0}=Y$, then the out put becomes zero through out the time interval and hence $X_{0}$ can not be determined in this case from the knowledge of $Z(t)$. It is a contradiction to our assumption. Thus the observability Gramian matrix $G_{o}\left(t_{0}, t_{f}\right)$ is invertible.

Now, in the next two theorems, the stability properties of the system (1) are discussed.

Definition 3.3:([7, 16]) The system (1) is called stable if for any $\varepsilon>0$ and $t_{0} \geq 0$, there exists $\delta=\delta\left(t_{0}, \varepsilon\right)>0$ such that for any solution $\bar{X}(t)=X\left(t, t_{0}, \bar{X}_{0}\right) \quad$ of $(1), \quad\left|\bar{X}_{0}-X_{0}\right| \leq \delta$ implies $|\bar{X}(t)-X(t)|<\varepsilon$ for all $t \geq t_{0} \geq 0$.

Definition 3.4:([7, 16]) The system (1) is called uniformly stable if $\delta$ in definition 3.3 is independent of the time $t_{0}$.

Definition 3.5:([7, 16]) The system (1) is called asymptotically stable if it is stable and if there exists $\delta>0$ such that $\left|\bar{X}_{0}-X_{0}\right| \leq$ $\delta$ implies $|\bar{X}(t)-X(t)| \rightarrow 0$ as $t \rightarrow+\infty$.

Theorem 3.4: Assume that
(a) $\left|\Psi\left(t, t_{0}\right)\right| \leq S$
(b) $|H(\tau, X(\tau)) U(\tau)| \leq T|X(\tau)|$ 
where $S>0$ and $T>0$. Then the solution of (1) is uniformly stable.

Proof :

For any $\varepsilon>0$, let $\delta(\varepsilon)<\frac{\varepsilon}{\operatorname{Ses}_{S T}\left(t, t_{0}\right)}$ and $\left|X_{0}\right|<\delta(\varepsilon)$. Suppose that there exists $t_{1} \geq t_{0}$ such that $\left|X\left(t_{1}\right)\right|=\varepsilon$ and $|X(t)|<\varepsilon$ on $\left[t_{0}, t_{1}\right)$

From (2), we have

$$
\begin{aligned}
X(t)= & \Psi\left(t, t_{0}\right) X_{0} \\
& +\Psi\left(t, t_{0}\right) \int_{t_{0}}^{t} \Psi\left(t_{0}, \sigma(\tau)\right)\left(\int_{t_{0}}^{\tau} K(\tau, \mathrm{s}, X(s)) \Delta s+B(\tau)\right) \\
& \times U(\tau) \Delta \tau, \text { on }\left[t_{0}, t_{1}\right] \\
|X(t)| \leq & \left|\Psi\left(t, t_{0}\right)\right|\left|X_{0}\right|+\int_{t_{0}}^{t}|\Psi(t, \sigma(\tau))||H(\tau, X(\tau))||U(\tau)| \Delta \tau \\
\leq & S \delta(\varepsilon)+S \int_{t_{0}}^{t} T|X(\tau)| \Delta \tau
\end{aligned}
$$

By Lemma 2.9, we have

$$
\begin{aligned}
|X(t)| & \leq S \delta(\varepsilon) e_{S T}\left(t, t_{0}\right) \\
& <S e_{S T}\left(t, t_{0}\right) \frac{\varepsilon}{S e_{S T}\left(t, t_{0}\right)} \\
& =\varepsilon
\end{aligned}
$$

Therefore $\left|X\left(t_{1}\right)\right|<\varepsilon$, for $t \in\left[t_{0}, t_{1}\right]$ which is a contradiction Thus the solution of (1) is uniformly stable.

Theorem 3.5: Assume that

(a) $\mid \Psi\left(t, t_{0}\right)\left\|X_{0}\right\| \leq S e_{P}\left(t_{0}, t\right)$,

(b) $\left|\Psi\left(t, t_{0}\right) \Psi(t, \sigma(\tau))\right| \leq T e_{P}(0, t)$

(c)

$$
\begin{gathered}
\sup _{t_{0} \leq s \leq \tau<\infty}\left(\int_{t_{0}}^{\tau}|K(\tau, s, X(\tau))| \Delta \tau+|B(\tau)|\right)|U(\tau)| \\
\leq L e_{P}(s, 0)|X(s)|
\end{gathered}
$$

where $S>0, T>0, L>0$ and $(P \ominus L T)>0$. Then every solution $X(t)$ of (1) tends to zero, as $t \rightarrow+\infty$.

\section{Proof :}

From (2), we have

$$
\begin{aligned}
X(t)= & \Psi\left(t, t_{0}\right) X_{0} \\
+ & \Psi\left(t, t_{0}\right) \int_{t_{0}}^{t} \Psi\left(t_{0}, \sigma(\tau)\right)\left(\int_{t_{0}}^{\tau} K(\tau, s, X(s)) \Delta s+B(\tau)\right) \\
& \times U(\tau) \Delta \tau, \\
|X(t)| \leq & \left|\Psi\left(t, t_{0}\right)\right|\left|X_{0}\right| \\
& +\left|\Psi\left(t, t_{0}\right)\right| \int_{t_{0}}^{t}\left|\Psi\left(t_{0}, \sigma(\tau)\right)\right| \\
& \times\left(\int_{t_{0}}^{\tau}|K(\tau, \mathrm{s}, X(s))| \Delta s+|B(\tau)|\right)|U(\tau)| \Delta \tau \\
\leq & S e_{P}\left(t_{0}, t\right)+L T e_{P}(0, t) \int_{t_{0}}^{t} e_{P}(s, 0)|X(s)| \Delta s \\
= & S e_{P}\left(t_{0}, 0\right) e_{P}(0, t)+L T e_{P}(0, t) \int_{t_{0}}^{t} e_{P}(s, 0)|X(s)| \Delta s
\end{aligned}
$$

$|X(t)| e_{P}(t, 0) \leq S e_{P}\left(t_{0}, 0\right)+L T \int_{t_{0}}^{t} e_{P}(s, 0)|X(s)| \Delta s$
By Lemma 2.9, we have

$$
\begin{aligned}
|X(t)| e_{P}(t, 0) & \leq S e_{P}\left(t_{0}, 0\right) e_{L T}\left(t, t_{0}\right) \\
& =S e_{P}\left(t_{0}, 0\right) e_{P}(0, t) e_{L T}\left(t, t_{0}\right) \\
& =S e_{P}\left(t_{0}, 0\right) e_{P}(0, t) e_{\ominus L T}\left(t_{0}, t\right) \\
& =S e_{P}\left(t_{0}, 0\right) e_{P}(0, t) e_{\ominus L T}\left(t_{0}, 0\right) e_{\ominus L T}(0, t) \\
& =S e_{P \ominus R T}\left(t_{0}, 0\right) e_{P \ominus L T}(0, t) .
\end{aligned}
$$

By Lemma 2.10, we have

$$
e_{P \ominus L T}(0, t) \leq \frac{1}{1+(P \ominus L T) t} \text {, so we obtain }
$$

$$
|X(t)| \leq S e_{P \ominus L T}\left(t_{0}, 0\right) \frac{1}{1+(P \ominus L T) t}
$$

Since $(P \ominus L T)>0$, we obtain the required result.

Corollary: Suppose $K(t, s, X(s))=K(t, s) X(s)$, then the solution of (1) is

$$
\begin{aligned}
X(t)= & \Psi\left(t, t_{0}\right) X_{0} \\
& + \\
& \Psi\left(t, t_{0}\right) \int_{t_{0}}^{t} \Psi\left(t_{0}, \sigma(\tau)\right)\left(\int_{t_{0}}^{\tau} K(\tau, s) X(s) \Delta s+B(\tau)\right) \\
& \times U(\tau) \Delta \tau
\end{aligned}
$$

\section{Example}

Example 4.1: Let us consider the following system on time scale

$$
\begin{aligned}
& X^{\Delta}(t)=\left[\begin{array}{ll}
0 & 1 \\
0 & 0
\end{array}\right] X(t)+\left(\int_{0}^{t}\left[\begin{array}{ll}
1 & 0 \\
0 & 1
\end{array}\right] X(s) \Delta s+\left[\begin{array}{ll}
1 & 1 \\
1 & 1
\end{array}\right]\right) U(t) \\
& Z(t)=\left[\begin{array}{ll}
1 & 1 \\
1 & 1
\end{array}\right] X(t) \\
& X(0)=\left[\begin{array}{ll}
1 & 1 \\
1 & 1
\end{array}\right]
\end{aligned}
$$

for $0 \leq s \leq t<\infty$.

Case (a): If $\mathbb{T}=\mathbb{R}^{+}$then (4) is

$$
\begin{aligned}
X^{\prime}(t) & =\left[\begin{array}{ll}
0 & 1 \\
0 & 0
\end{array}\right] X(t)+\left(\int_{0}^{t}\left[\begin{array}{ll}
1 & 0 \\
0 & 1
\end{array}\right] X(s) d s+\left[\begin{array}{ll}
1 & 1 \\
1 & 1
\end{array}\right]\right) U(t), \\
Z(t) & =\left[\begin{array}{ll}
1 & 1 \\
1 & 1
\end{array}\right] X(t), \\
X(0) & =\left[\begin{array}{ll}
1 & 1 \\
1 & 1
\end{array}\right] .
\end{aligned}
$$

The controllable Gramian matrix is given by

$G_{c}\left(t_{0}, t_{f}\right)=\left[\begin{array}{ll}1 / 2\left(-5+e^{2 t_{f}}\left(5+2\left(-3+t_{f}\right) t_{f}\right)\right) & 1 / 2\left(-3+e^{2 t_{f}}\left(3-2 t_{f}\right)\right) \\ 1 / 2\left(-3+e^{2 t_{f}}\left(3-2 t_{f}\right)\right) & -1+e^{2 t_{f}}\end{array}\right]$.

Clearly $G_{c}\left(t_{0}, t_{f}\right)$ is symmetric and invertible when $t_{0}=0$ and $t_{f} \neq 0$. By using Theorem 3.2, it implies that system (5) is controllable.

The observable Gramian matrix is given by

$$
G_{o}\left(t_{0}, t_{f}\right)=\left[\begin{array}{ll}
2 t_{f} & 2 t_{f}+t_{f}^{2} \\
2 t_{f}+t_{f}^{2} & 2 t_{f}+2 t_{f}^{2}+2 t_{f}^{3} / 3
\end{array}\right] .
$$

Clearly $G_{o}\left(t_{0}, t_{f}\right)$ is symmetric and invertible when $t_{0}=0$ and $t_{f} \neq 0$. By using Theorem 3.3, it implies that system (5) is observable.

The solution of (5) is 


$$
\begin{aligned}
X(t) & =\left[\begin{array}{ll}
x_{11}(t) & x_{12}(t) \\
x_{21}(t) & x_{22}(t)
\end{array}\right] \\
& =\left[\begin{array}{ll}
\frac{e^{4}(3-5 t)+e^{8}(1+t)}{\left(1-18 e^{4}+e^{8}\right)} & \frac{e^{4}(3-5 t)+e^{8}(1+t)}{\left(1-18 e^{4}+e^{8}\right)} \\
+\frac{3 e^{4+2 t}(-7+3 t)+e^{2 t}(1+3 t)}{\left(1-18 e^{4}+e^{8}\right)} & +\frac{3 e^{4+2 t}(-7+3 t)+e^{2 t}(1+3 t)}{\left(1-18 e^{4}+e^{8}\right)} \\
1+\frac{-1+13 e^{4}}{\left(1-18 e^{4}+e^{8}\right)} & 1+\frac{-1+13 e^{4}}{\left(1-18 e^{4}+e^{8}\right)} \\
+\frac{e^{2 t}\left(1+2 t+e^{4}(-13+6 t)\right)}{\left(1-18 e^{4}+e^{8}\right)} & +\frac{e^{2 t}\left(1+2 t+e^{4}(-13+6 t)\right)}{\left(1-18 e^{4}+e^{8}\right)}
\end{array}\right] .
\end{aligned}
$$

It can be observed that $x_{11}(t)=x_{12}(t)$ and $x_{21}(t)=x_{22}(t)$ and which are plotted in the interval $[0,2]$ and are shown in figure 1.

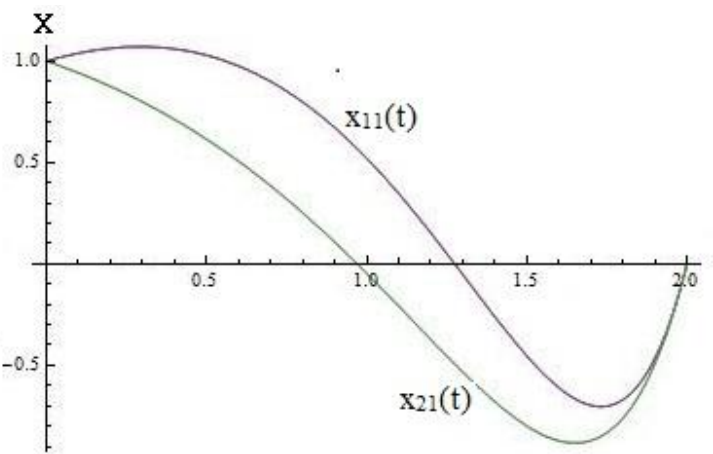

Figure 1:: Solution of (5)

From the figure 1 , it can be observe that for initial time $t_{0}=0$, initial state $X\left(t_{0}\right)=1$ and final state $X_{f}=0$, then there exists a finite final time $t_{f}=2$ such that $X\left(t_{f}\right)=X_{f}$. Hence the system (5) is controllable and observable from the definitions 3.1 and 3.2 .

Case (b): If $\mathbb{T}=\mathbb{N}$ then (4) is

$$
\left.\begin{array}{rl}
\Delta X(n)= & {\left[\begin{array}{ll}
0 & 1 \\
0 & 0
\end{array}\right] X(n)} \\
& +\left(\sum_{s=0}^{n-1}\left[\begin{array}{ll}
1 & 0 \\
0 & 1
\end{array}\right] X(s)+\left[\begin{array}{ll}
1 & 1 \\
1 & 1
\end{array}\right]\right) U(n) \\
Z(n)= & {\left[\begin{array}{ll}
1 & 1 \\
1 & 1
\end{array}\right] X(n),} \\
X(0)= & {\left[\begin{array}{ll}
1 & 1 \\
1 & 1
\end{array}\right] .}
\end{array}\right\}
$$

The controllable Gramian matrix is given by

$$
\begin{aligned}
& G_{c}\left(n_{0}, n_{f}\right) \\
& =\left[\begin{array}{ll}
\frac{2}{27}\left[-20+5\left(2^{2+2 n_{f}}\right)\right. & -\frac{2}{9}\left[4-2^{2+2 n_{f}}\right. \\
\left.-3\left(2^{3+2 n_{f}} n_{f}\right)+9\left(2^{2 n_{f}} n_{f}^{2}\right)\right] & -\frac{2}{9}\left[4-2^{2+2 n_{f}}+3\left(2^{2 n_{f}} n_{f}\right)\right] \\
-\frac{2}{9}\left[4-2^{2+2 n_{f}}+3\left(2^{2 n_{f}} n_{f}\right)\right] & \frac{2}{3}\left[-1+2^{2 n_{f}}\right]
\end{array}\right] .
\end{aligned}
$$

Clearly $G_{c}\left(n_{0}, n_{f}\right)$ is symmetric and invertible when $n_{0}=0$ and $n_{f} \neq 0$. By using Theorem 3.2, it implies that system (6) is controllable.

The observable Gramian matrix is given by

$$
G_{o}\left(n_{0}, n_{f}\right)=\left[\begin{array}{ll}
2 n_{f} & \left(1+n_{f}\right) n_{f} \\
n_{f}\left(1+n_{f}\right) & \frac{1}{3} n_{f}\left(1+n_{f}\right)\left(1+2 n_{f}\right)
\end{array}\right] .
$$

Clearly $G_{o}\left(n_{0}, n_{f}\right)$ is symmetric and invertible when $n_{0}=0$ and $n_{f} \neq 0$. By using Theorem 3.3, it implies that system (6) is observable.

The solution of $(6)$ is

$$
\begin{aligned}
X(n) & =\left[\begin{array}{ll}
x_{11}(n) & x_{12}(n) \\
x_{21}(n) & x_{22}(n)
\end{array}\right] \\
& =\left[\begin{array}{ll}
1 / 9\left[32-23\left(4^{n}\right)\right. & 1 / 9\left[32-23\left(4^{n}\right)\right. \\
\left.+3\left(8+3\left(4^{n}\right)\right) n\right] & \left.+3\left(8+3\left(4^{n}\right)\right) n\right] \\
8 / 3-5\left(4^{n}\right) / 3 & 8 / 3-5\left(4^{n}\right) / 3 \\
+3\left(4^{-1+n}\right) n & +3\left(4^{-1+n}\right) n
\end{array}\right] .
\end{aligned}
$$

It can be observed that $x_{11}(n)=x_{12}(n)$ and $x_{21}(n)=x_{22}(n)$ are plotted in the interval $[0,2]$ and are shown in figure 2 .

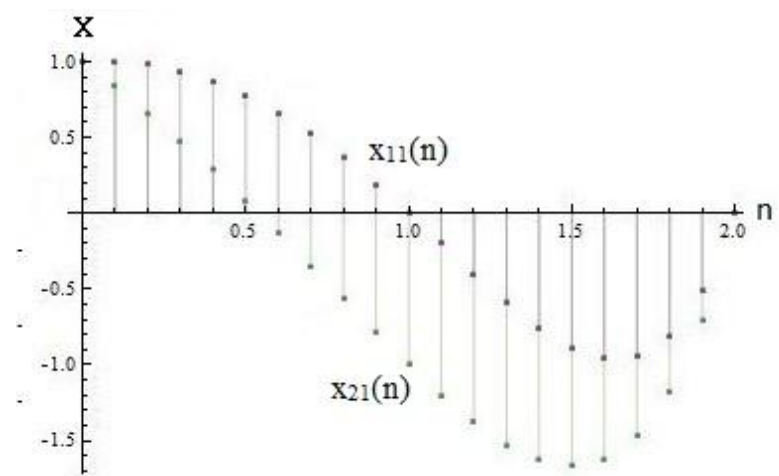

Figure 2: Solution of (6)

From the figure 2 , it can be observe that for initial time $n_{0}=0$, initial state $X\left(n_{0}\right)=1$ and final state $X_{f}=0$, then there exists a finite final time $n_{f}=2$ such that $X\left(n_{f}\right)=X_{f}$. Hence the system (6) is controllable and observable from the definitions 3.1 and 3.2 .

\section{Conclusion}

In this paper, the necessary and sufficient conditions for the controllability and observability of the solution of the system (1) are established. The results are demonstrated using examples. Graphically, the idea of controllability and observability for the given problem is illustrated in figures 1 and 2 .

\section{References}

[1] M. Adivar, "Principal matrix solution and variation of parameters for Volterra integro-dynamic equations on time scales", Glasg. Math. J, Vol.53, (2011), pp.463-480.

[2] L.C. Becker, "Principal matrix solutions and variation of parameters for a for Volterra-integro-differential equation and its adjoint", Electron J Qual Theory Differ Equ, Vol.14, (2006), pp.1-22.

[3] M. Bohner, A. Peterson, Dynamic Equations on Time Scales, Birkhauser, Boston, (2001).

[4] T.A. Burton, W.E. Mahfound, "Stability criteria for Volterra equations",Trans Am Math Soc Vol.279, (1983), pp.143-174.

[5] T.A. Burton, W.E. Mahfound, "Stability by decompositions for Volterra equations", Tohoku Math J JI Ser Vol.37, (1985), pp.489-511.

[6] T.A. Burton, Volterra integral and differential equations, 2nd edn. Elsevier, (2005)

[7] J.M. Davis, I.A. Gravagne, B.J.Jackson, R.J. Marks, "Controllability, observability, realizability, and stability of dynamic linear systems", Electron J Differ Equ Vol.37, (2009), pp.1-32.

[8] S. Hilger, Ein Mabkettenkalkul mit Anwendung auf Zentrumsmannigfaltigkeiten, $\mathrm{PhD}$ thesis, Universitat Wurzburg, (1988). 
[9] IA. Gravagne, JM. Devis and JJ. Dacunha, "A unified approach to high-gain adaptive controllers", J.Abstr Appl Anal, (2009).

[10] IA. Gravagne, JM. Devis and JJ. Dacunha, RJ II. Marks, "Bandwidth reduction for controller area networks using adaptive sampling", New Orleans, (2004), pp.5250-5255.

[11] IA. Gravagne, JM. Devis and RJ II. Marks, "How deterministic must a real time controller",Alberta, (2005), pp.3856-3861.

[12] R.E. Kalman, "On the general theory of control system",Proc.1st IFAC Congress Automatic control Vol.1, (1960), pp.481-492.

[13] R.E. Kalman, Y.C. Ho, K.S. Narendra, "Controllability of linear dynamical systems", Contrib. Differ. Equ Vol.1, (1963), pp.189-213.

[14] RJ II. Marks, IA. Gravagne, JM. Devis and JJ. Dacunha, "Nonregressivity in switching linear circuits and mechanical systems",Math Comput Model Vol.43, (2006), p.1383-1392.

[15] J. Seiffert, S. Sanyal, DC. Wunsch,"Hamilton-Jacobi -Bellman equations and approximate dynamic programming on time scales",IEEE Trans Syst Man Cybern Vol.38, (2008), pp.918-923.

[16] A. Yonus, Ghaus ur Rahman, "Controllability, Observability, and Stability of a Volterra integro-dynamic system on time scales",IEEE Trans Syst Man Cybern Vol.20, (2014), pp.383-402. 